\title{
Longitudinal wakefield for an axisymmetric collimator
}

\author{
A. Blednykh and S. Krinsky \\ Brookhaven National Laboratory, Upton, New York 11973, USA
}

(Received 16 March 2012; published 25 May 2012)

\begin{abstract}
We consider the longitudinal point-charge wakefield, $w(s)$, for an axisymmetric collimator having inner radius $b$, outer radius $d$, inner length $g$, and taper length $L$. The taper angle $\alpha$ is defined by $\tan \alpha=$ $(d-b) / L$. Using the electromagnetic simulation code ECHO, we explore the dependence of the wakefield on a collimator's geometric parameters over a wide range of profiles: from small-angle tapers to stepfunction transitions. The point-charge wakefield is determined using an approximation introduced by Podobedov and Stupakov. We have found it useful to exhibit the wakefield as a function of the scaled variable $s / d \alpha$. For small taper angles, our results illustrate the satisfaction of the longitudinal scaling found by Stupakov, Bane, and Zagorodnov; and for larger taper angles, the breaking of this longitudinal scaling is clearly depicted. The use of the scaled variable $s / d \alpha$ turns out to be especially well suited to describing the wakefield for a collimator with step-function profile $(\alpha=\pi / 2)$.
\end{abstract}

DOI: 10.1103/PhysRevSTAB.15.054405

PACS numbers: 41.20.Jb, 41.60.-m

\section{INTRODUCTION}

Knowledge of wakefields is of critical importance for determining the current-dependent collective effects limiting performance in high-intensity accelerators. In recent years, there has been considerable effort to understand the properties of the short-range wakefield generated when a short bunch passes through a collimating structure. Progress in understanding this subject has been achieved through both analytical and numerical studies. In this paper, using the $2 \mathrm{D}$ electromagnetic simulation ECHO code $[1,2]$, we carry out an investigation of the longitudinal short-range wakefield due to an axisymmetric collimator.

We consider the highly relativistic limit in which a particle traveling at the speed of light $c$, passes through a step function or tapered collimator illustrated in Figs. 1(a) and 1(b). We denote the smaller pipe radius by $b$, the larger radius by $d$, the length of the inner section of the collimator by $g$, and the length of the taper by $L$. The taper angle $\alpha$ is defined by $\tan \alpha=(d-b) / L$. The longitudinal wakefield produced by a point charge is denoted $w(s)$, where $s$ is the distance of the test particle behind the driving particle.

Podobedov and Stupakov [3,4] have noted that the pointcharge wakefield for a collimator can be written in the form

$$
w(s ; b, d, g, L)=\frac{c Z_{0}}{\pi} \log (d / b) \delta(s)+D(s ; b, d, g, L),
$$

where $Z_{0}$ is the impedance of free space. The delta function term corresponds to the result in the optical regime [5]. The causal function $D(s)$ vanishes for $s<0$ and is discontinuous

Published by the American Physical Society under the terms of the Creative Commons Attribution 3.0 License. Further distribution of this work must maintain attribution to the author(s) and the published article's title, journal citation, and DOI. at $s=0$. Since the impedance vanishes at zero frequency, it follows that

$$
\int_{0}^{\infty} d s D(s)=-\frac{c Z_{0}}{\pi} \log (d / b)
$$

In this paper, in order to facilitate the illustration of the behavior of the wakefield over a wide range of parameters and for larger $s$, we prefer to introduce the normalized causal function $u(s)$, via

$w(s ; b, d, g, L)=\frac{c Z_{0}}{\pi} \log (d / b)[\delta(s)-u(s ; b, d, g, L)]$,

where

$$
\int_{0}^{\infty} d s u(s)=1
$$

Using this normalized function, the ratio of the loss factor $k_{\text {loss }}$ to that in the optical regime [5,6] $k_{\text {loss }}^{\text {opt }}$ is easily expressed. For a Gaussian bunch of rms width $\sigma$, this ratio is given by

$$
k_{\text {loss }}(\sigma) / k_{\text {loss }}^{\mathrm{opt}}(\sigma)=1-\int_{0}^{\infty} d s u(s) \exp \left(-s^{2} / 4 \sigma^{2}\right),
$$

where $k_{\mathrm{loss}}^{\mathrm{opt}}(\sigma)=\left(c Z_{0} / 2 \pi^{3 / 2} \sigma\right) \log (d / b)$.

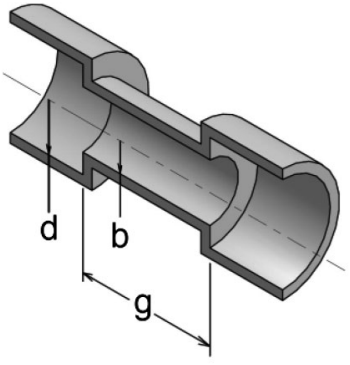

a)

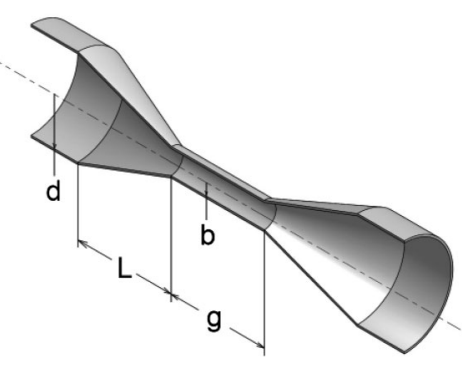

b)
FIG. 1. Step collimator (a) and tapered collimator (b). 
For a small-angle tapered collimator (sat), it is shown in Ref. [4] that

$$
D^{s a t}(0) \cong-\frac{c Z_{0}}{\pi b \alpha},
$$

and for a step collimator $(s t)$, it follows from the work of Okamoto, Jiang, and Gluckstern [7] that

$$
D^{s t}(0) \cong-\frac{0.6 c Z_{0}}{\pi b(\pi / 2)}
$$

We now note that, to within $\pm 10 \%$ accuracy,

$$
\frac{1}{\pi b} \cong \frac{\log (d / b)}{d}, \quad \text { for } 1.5 \preceq d / b \preceq 6 .
$$

In this paper, we shall restrict our attention to collimators with parameters within the range $1.5 \preceq d / b \preceq 6$. In this case, Eqs. (6) and (8) imply that, for small-angle tapers,

$$
u^{\text {sat }}(0) \cong \frac{\pi}{d \alpha},
$$

and Eqs. (7) and (8) show that for a step collimator,

$$
u^{s t}(0) \cong \frac{0.6 \pi}{d(\pi / 2)} .
$$

The short-range wakefield depends predominantly on the two length scales $b \alpha$ and $d \alpha$. In illustrating its behavior, one can plot the wakefield versus the variable $s / b \alpha$ or $s / d \alpha$. In this paper, in order to illustrate more clearly the behavior of the wakefield at longer distances, we prefer to utilize the variable $s / d \alpha$.

For the short-range wakefield $(s / d \alpha \lesssim 0.3)$, our numerical calculations demonstrate that the dependence on $g$ is very weak and they support the approximate validity of the scaling relation,

$$
w(s ; b, d, g, L) \cong \frac{c Z_{0}}{\pi} \log \left(\frac{d}{b}\right)\left[\delta(s)-\frac{1}{d \alpha} f\left(\frac{s}{d \alpha}, \frac{b}{d}\right)\right] .
$$

With the wakefield approximated by Eq. (11), it follows from Eq. (5) that the departure of the loss factor from the optical approximation for short bunches $(\sigma / d \alpha \lesssim 0.15)$ can be estimated by

$$
k_{\mathrm{loss}}(\sigma) / k_{\mathrm{loss}}^{\mathrm{opt}}(\sigma) \cong 1-\sqrt{\pi} f\left(0, \frac{b}{d}\right) \frac{\sigma}{d \alpha},
$$

with $f\left(0, \frac{b}{d}\right)$ varying from $\sim 3$ for small-angle tapers to $\sim 1.8$ for step collimators. Equation (12) is a more accurate expression for the loss factor than the similar relation given in Eq. (12.26) of Ref. [8].

\section{DIMENSIONAL ANALYSIS}

We motivate our discussion by using dimensional analysis and the longitudinal scaling relation introduced in Ref. [9]. From dimensional analysis, we can write

$$
u(s ; b, d, g, L)=\frac{1}{d} u_{1}\left(\frac{s}{d} ; \frac{b}{d}, \frac{g}{d}, \frac{L}{d}\right) .
$$

In the special case of a step collimator, for which $L=0$,

$$
u^{s t}(s ; b, d, g)=\frac{1}{d} u_{1}^{s t}\left(\frac{s}{d} ; \frac{b}{d}, \frac{g}{d}, 0\right) .
$$

We have determined that when $g$ is not too short (see Fig. 2), the wakefield depends very weakly on $g$, so we can simplify Eq. (14) to read

$$
u^{s t}(s ; b, d, g) \cong \frac{1}{d} u_{2}^{s t}\left(\frac{s}{d} ; \frac{b}{d}\right) .
$$

The corresponding weak dependence of the impedance of a step collimator on $g$ was found in the analysis of Ref. [7].

Our ECHO calculations have uncovered the surprising result that in the parameter range we are considering [see Eq. (8)], and for $s / d \geqslant 0.5$, the dependence on $b / d$ is very weak (see Fig. 3), so to a reasonable approximation,

$$
u^{s t}(s ; b, d, g) \cong \frac{1}{d} u_{3}^{s t}\left(\frac{s}{d}\right) .
$$

Clearly, in Eq. (13), we could have chosen to set the length scale with any of the lengths in the problem. Equation (16) provides one motivation for our choice to single out the dependence on the larger radius $d$.

For a small-angle tapered collimator, it has been shown [9] that an important longitudinal scaling relation holds for the short-range wakefield,

$$
w^{\mathrm{sat}}(s ; b, d, g, L)=\lambda w^{\mathrm{sat}}\left(s \lambda ; b, d, \frac{g}{\lambda}, \frac{L}{\lambda}\right),
$$

where $\lambda$ is a dimensionless parameter. The scaling relation (17) holds trivially for the delta function term in Eq. (3). It therefore follows from Eqs. (13) and (17) that

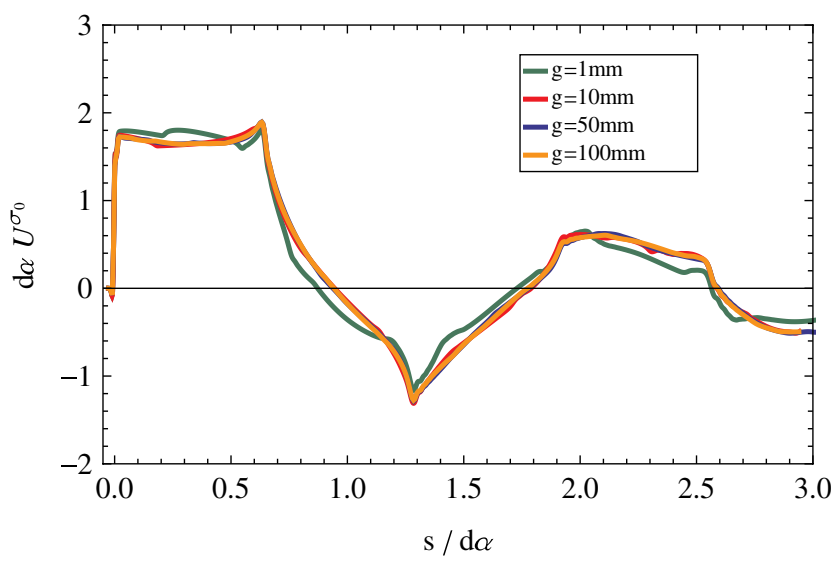

FIG. 2. The function $f \cong d \alpha U^{\sigma_{0}}$ versus $s / d \alpha$ for step collimators $(\alpha=\pi / 2)$ with $d=6 \mathrm{~mm}, b=3 \mathrm{~mm}, \sigma_{0}=50 \mu \mathrm{m}$ and $g=1 \mathrm{~mm}, g=10 \mathrm{~mm}, 50 \mathrm{~mm}$, and $100 \mathrm{~mm}$. 


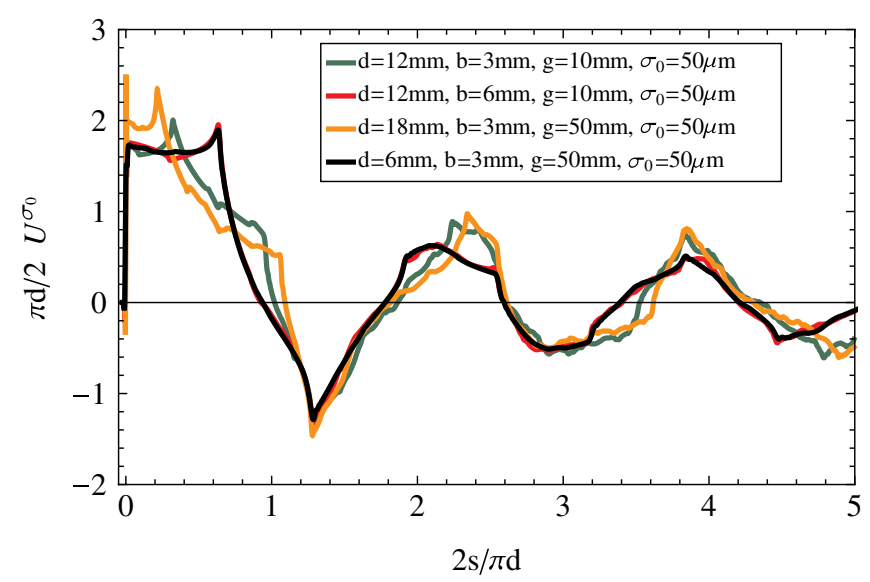

FIG. 3. For step collimator, we plot $(\pi d / 2) U^{\sigma_{0}}(s ; b, d, g)$ versus $2 s / \pi d$ for $\sigma_{0}=50 \mu \mathrm{m}$ and $d=12 \mathrm{~mm}, b=3 \mathrm{~mm}$, $g=10 \mathrm{~mm}$ (green); $d=12 \mathrm{~mm}, b=6 \mathrm{~mm}, g=10 \mathrm{~mm}$ (red); $d=18 \mathrm{~mm}, b=3 \mathrm{~mm}, g=50 \mathrm{~mm}$ (orange); and $d=$ $6 \mathrm{~mm}, b=3 \mathrm{~mm}, g=50 \mathrm{~mm}$ (black).

$$
u_{1}^{\mathrm{sat}}\left(\frac{s}{d} ; \frac{b}{d}, \frac{g}{d}, \frac{L}{d}\right)=\lambda u_{1}^{\mathrm{sat}}\left(\frac{s \lambda}{d} ; \frac{b}{d}, \frac{g}{\lambda d}, \frac{L}{\lambda d}\right) .
$$

We now choose $\lambda=L /(d-b)$, obtaining

$u_{1}^{\mathrm{sat}}\left(\frac{s}{d} ; \frac{b}{d}, \frac{g}{d}, \frac{L}{d}\right)=\frac{L}{d-b} u_{1}^{\mathrm{sat}}\left(\frac{s L}{d(d-b)} ; \frac{b}{d}, \frac{g(d-b)}{L d}, \frac{d-b}{d}\right)$,

and, hence,

$u^{\mathrm{sat}}(s ; b, d, g, L)=\frac{L}{d(d-b)} u_{2}^{\mathrm{sat}}\left(\frac{s L}{d(d-b)} ; \frac{b}{d}, \frac{g(d-b)}{L d}\right)$.

Recall that the taper angle $\alpha$ is defined by $\tan \alpha=$ $(d-b) / L$. We can rewrite Eq. (20) in the form

$$
u^{\mathrm{sat}}(s ; b, d, g, L)=\frac{1}{d \tan \alpha} u_{2}^{\mathrm{sat}}\left(\frac{s}{d \tan \alpha} ; \frac{b}{d}, \frac{g \tan \alpha}{d}\right) .
$$

Based on the ECHO calculations, we have found that the dependence on $g$ is weak for $s / d \alpha \lesssim 0.3$. Neglecting the dependence on $g$, and replacing $\tan \alpha$ by the angle $\alpha$ in the first argument of $u_{2}^{\text {sat }}$, we obtain the approximate scaling relation:

$$
u^{\mathrm{sat}}(s ; b, d, g, L) \cong \frac{1}{d \alpha} u_{2}^{\mathrm{sat}}\left(\frac{s}{d \alpha} ; \frac{b}{d}\right) .
$$

Let us note that Eq. (22), which we have just introduced for the tapered collimator, has a form consistent with Eq. (15) found for the step collimator $(\alpha=\pi / 2)$. Therefore, we can hope that Eq. (15) will have an approximate validity for large collimator angles. Our numerical calculations show that replacing the tangent by the angle extends the range of usefulness of Eq. (15). In the context of the derivation of the scaling relation of Ref. [9], replacing $\tan \alpha$ by the angle $\alpha$ corresponds to moving out of the region of validity of the paraxial approximation, which is only applicable for a smooth, slowly varying wall. Once the taper angle approaches $\pi / 2$, the scaling is broken and the function $f$ begins to depend also on the angle $\alpha$ itself. The special case of the wakefield of the step collimator ( $\alpha=\pi / 2)$ turns out to exhibit very interesting behavior deserving individual attention.

\section{APPROXIMATE CALCULATION OF THE POINT-CHARGE WAKEFIELD}

Using ECHO, we cannot directly calculate the pointcharge wakefield. In Refs. [3,4], an approximate method for determining the point-charge wake has been introduced. Following this approach, we use ECHO to calculate the wakefield $W^{\sigma_{0}}(s)$ produced by a Gaussian bunch of rms width $\sigma_{0}$. We choose $\sigma_{0}$ small enough so that the wake is resistive. We then approximate Eq. (3) by

$$
\begin{aligned}
W^{\sigma_{0}}(s ; b, d, g, L)= & \frac{c Z_{0}}{\pi} \log (d / b)\left[\frac{1}{\sqrt{2 \pi} \sigma_{0}} \exp \left(\frac{-s^{2}}{2 \sigma_{0}^{2}}\right)\right. \\
& \left.-U^{\sigma_{0}}(s ; b, d, g, L)\right] .
\end{aligned}
$$

For $s \gg \sigma_{0}$, the point-charge wake is well approximated by Eq. (23), hence,

$$
u(s ; b, d, g, L) \cong U^{\sigma_{0}}(s ; b, d, g, L) \quad\left(s \gg \sigma_{0}\right) .
$$

In this manner, we approximate the scaling function introduced in Eq. (11) by

$$
f \cong d \alpha U^{\sigma_{0}}(s ; b, d, g, L) \quad\left(s \gg \sigma_{0}\right) .
$$

We shall now discuss the results of our ECHO calculations and illustrate the scaling of the short-range wakefield.

\section{A. Step collimators}

In Fig. 2, we plot the scaling function $f \cong d \alpha U^{\sigma_{0}}$ versus $s / d \alpha$ for step collimators $(\alpha=\pi / 2)$ with $d=6 \mathrm{~mm}, b=$ $3 \mathrm{~mm}, \sigma_{0}=50 \mu \mathrm{m}$ and $g=1 \mathrm{~mm}, g=10 \mathrm{~mm}, 50 \mathrm{~mm}$, and $100 \mathrm{~mm}$. We note that $f$ is quite independent of $g$. Only negligible variation is observed when $g$ varies from 100 to $10 \mathrm{~mm}$. Even at $g=1 \mathrm{~mm}$ the deviation is very small. In Fig. 3, we plot $f \cong d \alpha U^{\sigma_{0}}$ versus $s / d \alpha$ for step collimators with $b=3 \mathrm{~mm}$ and $d=6 \mathrm{~mm}$ (black), $12 \mathrm{~mm}$ (green) and $18 \mathrm{~mm}$ (orange), and with $b=6 \mathrm{~mm}$ and $d=$ $12 \mathrm{~mm}$ (red). The rms length of the charge distribution $\sigma_{0}=50 \mu \mathrm{m}$ is chosen to be small enough to assure that the wakefield is resistive. The close agreement of the wakefields for these different parameters is striking. These results clearly show that the dependence of the scaling function $f$ on the ratio $b / d$ is surprisingly weak, as noted in Eq. (16).

The scaled loss factor $k_{\text {loss }} / k_{\text {loss }}^{\text {opt }}$ as a function of $\sigma / d \alpha$ ( $\alpha=\pi / 2$ ) for several step collimators is illustrated in 


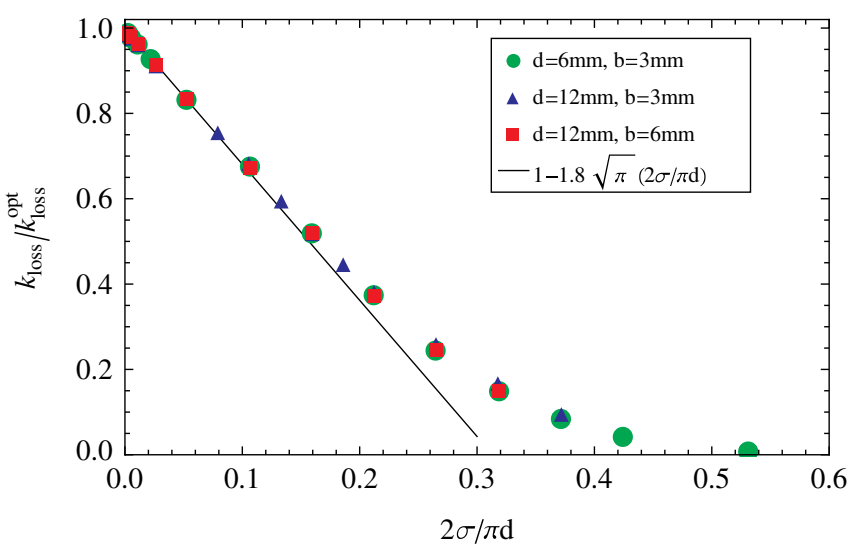

FIG. 4. We plot $k_{\text {loss }} / k_{\text {loss }}^{\text {opt }}$ versus $\sigma / d \alpha$ for $\alpha=\pi / 2$. The solid line [see Eq. (12)] is $k_{\text {loss }} / k_{\text {loss }}^{\text {opt }}=1-1.8 \sqrt{\pi}(\sigma / d \alpha)$. Round step $g=10 \mathrm{~mm}$.

Fig. 4. Good agreement is found with Eq. (12) for $2 \sigma / \pi d \lesssim 0.15$.

\section{B. Tapered collimators}

In Fig. 5, we plot the scaling function $f \cong d \alpha U^{\sigma_{0}}$ versus $s / d \alpha$ for tapered collimators with $d=6 \mathrm{~mm}, b=3 \mathrm{~mm}$, $L=6.25 \mathrm{~mm}, \sigma_{0}=30 \mu \mathrm{m}$ and $g=12.5 \mathrm{~mm}$ (green), $50 \mathrm{~mm}$ (aqua), and $100 \mathrm{~mm}$ (red). We note that for $s / d \alpha \lesssim$ $0.3, f$ is quite independent of $g$. In Fig. 6, we plot the function $f \cong d \alpha U^{\sigma_{0}}$ versus $s / d \alpha$ for collimators with $d=$ $6 \mathrm{~mm}$ and $b=3 \mathrm{~mm}$. The cases with taper length $L \gtrsim$ $6.25 \mathrm{~mm}$ (red, blue, orange) satisfy the longitudinal scaling (17) very accurately. The longitudinal scaling is seen to be broken when $L \preceq 3.125 \mathrm{~mm}$. The accuracy of the approximation of Eq. (11) for the cases of $L=3.12 \mathrm{~mm}$ (aqua) and $L=1.56 \mathrm{~mm}$ (green) has been significantly improved by the replacement of $\tan \alpha$ by the angle $\alpha$ as suggested following Eq. (21). (See Fig. 11 in the Appendix.) Even in the case of the step collimator (black),

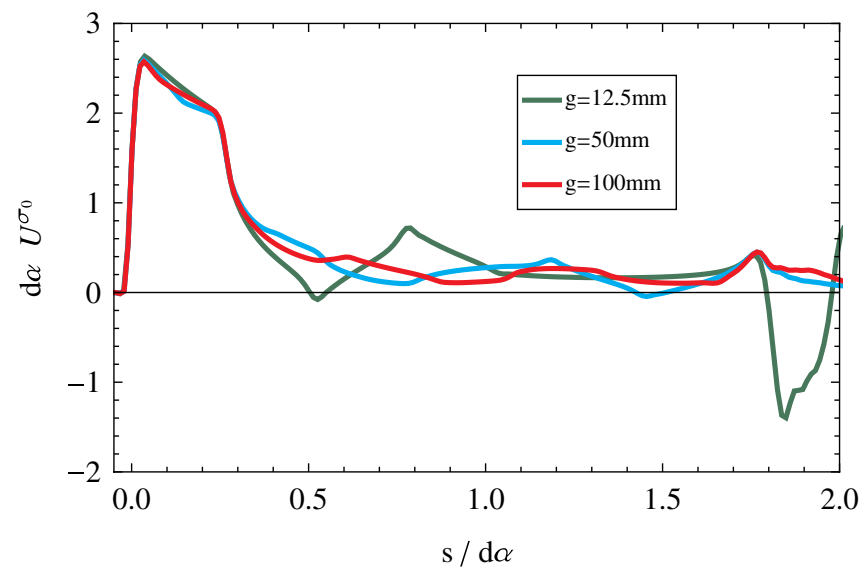

FIG. 5. The function $f \cong d \alpha U^{\sigma_{0}}$ versus $s / d \alpha$ for tapered collimators with $d=6 \mathrm{~mm}, b=3 \mathrm{~mm}, \sigma_{0}=30 \mu \mathrm{m}$ and $g=$ $12.5 \mathrm{~mm}, 50 \mathrm{~mm}$, and $100 \mathrm{~mm}$.

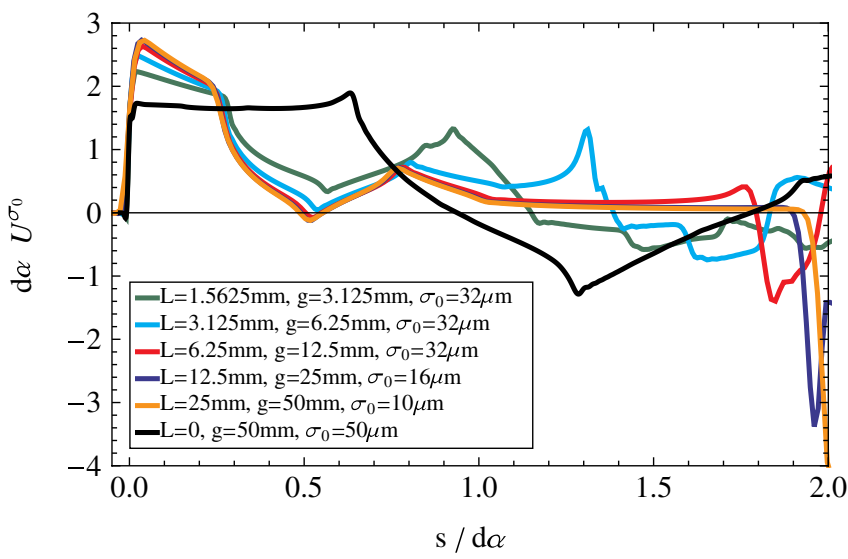

FIG. 6. For tapered collimators, we plot $d \alpha U^{\sigma_{0}}$ versus $s / d \alpha$ for $d=6 \mathrm{~mm}, b=3 \mathrm{~mm}$ and $g=3.12 \mathrm{~mm}, L=1.56 \mathrm{~mm}$, $\sigma_{0}=32 \mu \mathrm{m}$ (green); $g=6.25 \mathrm{~mm}, L=3.12 \mathrm{~mm}, \quad \sigma_{0}=$ $32 \mu \mathrm{m}$ (aqua); $g=12.5 \mathrm{~mm}, L=6.25, \sigma_{0}=32 \mu \mathrm{m}$ (red); $g=25 \mathrm{~mm}, L=12.5 \mathrm{~mm}, \sigma_{0}=16 \mu \mathrm{m}$ (blue); and $g=$ $50 \mathrm{~mm}, L=25 \mathrm{~mm}, \sigma_{0}=8 \mu \mathrm{m}$ (orange). The black curve corresponds to a step collimator. We see that longitudinal scaling is only accurately satisfied for $L \gtrsim 6.25 \mathrm{~mm}$.

Eq. (11) provides a rough approximation for small $s$. In Fig. 7, we plot the function $f \cong d \alpha U^{\sigma_{0}}$ versus $s / d \alpha$ for collimators with $d=12 \mathrm{~mm}$ and $b=3 \mathrm{~mm}$. The cases with taper length $L \gtrsim 12.5 \mathrm{~mm}$ (pink, black, orange) satisfy the longitudinal scaling (17) very accurately. The longitudinal scaling is seen to be broken for the cases of $L \lesssim 6.25 \mathrm{~mm}$ (red, aqua, green). In Fig. 8, we plot the function $f \cong d \alpha U^{\sigma_{0}}$ versus $s / d \alpha$ for collimators with $d=$ $6 \mathrm{~mm}, \quad b=3 \mathrm{~mm}, L=25 \mathrm{~mm}$ (red); $d=12 \mathrm{~mm}$, $b=3 \mathrm{~mm}, L=50 \mathrm{~mm}$ (black); and $d=18 \mathrm{~mm}, b=$ $3 \mathrm{~mm}, L=50 \mathrm{~mm}$ (green) for values of $L$ sufficiently

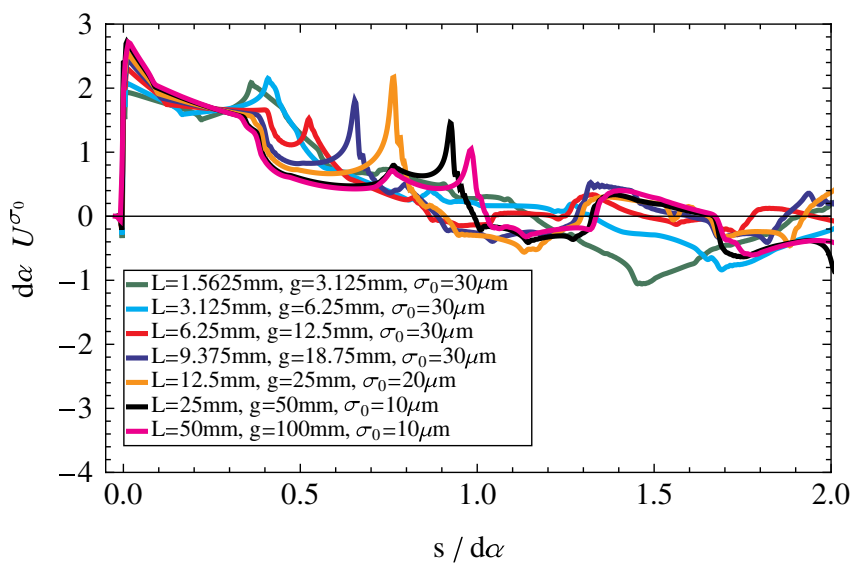

FIG. 7. For tapered collimators, we plot $d \alpha U^{\sigma_{0}}$ versus $s / d \alpha$ for $d=12 \mathrm{~mm}$ and $b=3 \mathrm{~mm}$ and $g=3.12 \mathrm{~mm}, L=$ $1.56 \mathrm{~mm}, \sigma_{0}=30 \mu \mathrm{m}$ (green); $g=6.25 \mathrm{~mm}, L=3.12 \mathrm{~mm}$, $\sigma_{0}=30 \mu \mathrm{m}$ (aqua); $g=12.5 \mathrm{~mm}, L=6.25, \sigma_{0}=30 \mu \mathrm{m}$ (red); $g=18.8 \mathrm{~mm}, L=9.4 \mathrm{~mm}, \sigma_{0}=30 \mu \mathrm{m}$ (blue); $g=$ $25 \mathrm{~mm}, L=12.5 \mathrm{~mm}, \sigma_{0}=20 \mu \mathrm{m}$ (orange); $d=12 \mathrm{~mm}$, $g=50 \mathrm{~mm}, L=25 \mathrm{~mm}, \sigma_{0}=10 \mu \mathrm{m}$ (black); and $g=$ $100 \mathrm{~mm}, L=50 \mathrm{~mm}, \sigma_{0}=10 \mu \mathrm{m}$ (purple). 


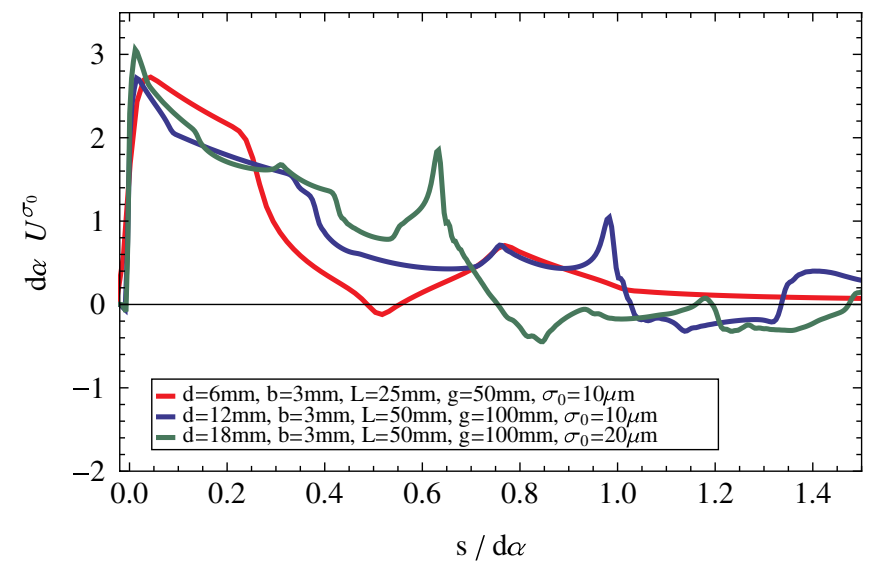

FIG. 8. For small-angle tapered collimators satisfying longitudinal scaling, we plot $d \alpha U^{\sigma_{0}}$ versus $s / d \alpha$ for $d=6 \mathrm{~mm}, b=$ $3 \mathrm{~mm}, L=25 \mathrm{~mm}$ (red); $d=12 \mathrm{~mm}, b=3 \mathrm{~mm}, L=50 \mathrm{~mm}$ (black); and $d=18 \mathrm{~mm}, b=3 \mathrm{~mm}, L=50 \mathrm{~mm}$ (green).
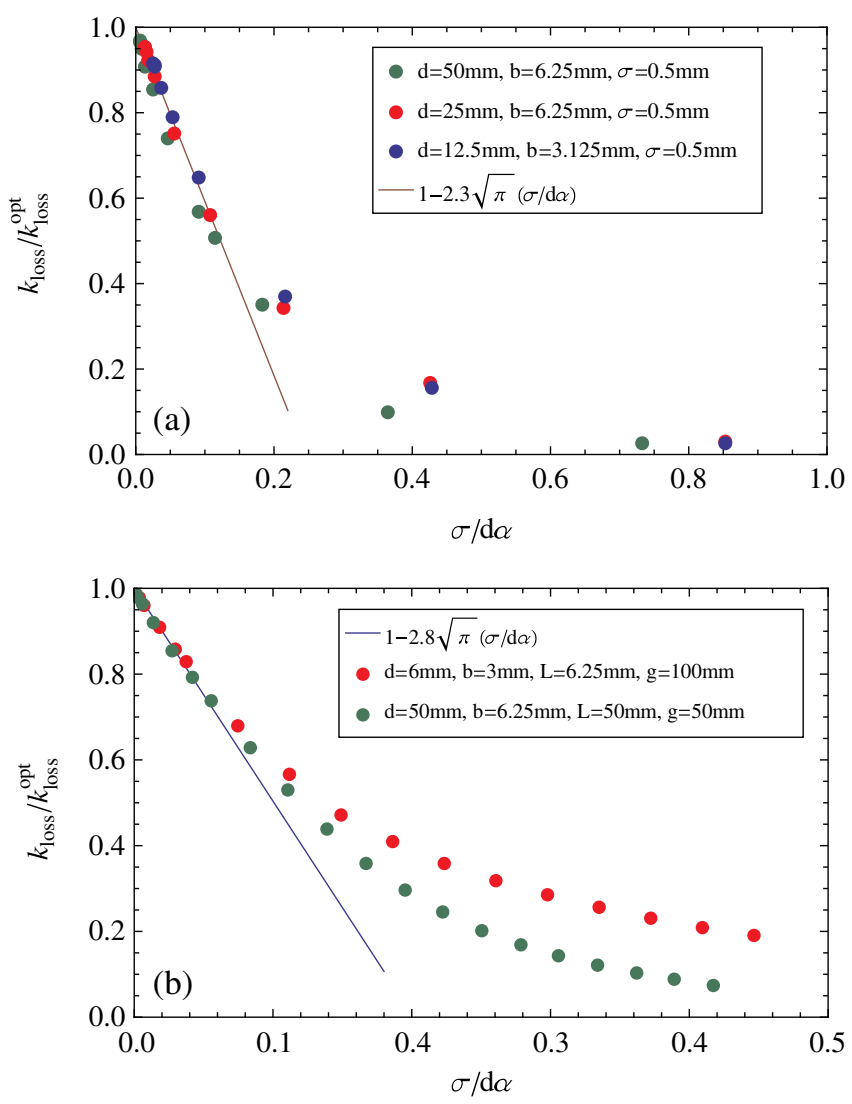

FIG. 9. For tapered collimators, we plot $k_{\text {loss }} / k_{\text {loss }}^{\text {opt }}$ versus $\sigma / d \alpha$. The solid line corresponds to Eq. (12). (a) Varied taper length $L$ for fixed bunch length $\sigma=0.5 \mathrm{~mm}$ for collimators with $d=50 \mathrm{~mm}, b=6.25 \mathrm{~mm}$ (green dots), $d=25 \mathrm{~mm}, b=$ $6.25 \mathrm{~mm}$ (red dots), $d=12.5 \mathrm{~mm}, b=3.125 \mathrm{~mm}$ (blue dots), and inner length $g=10 \mathrm{~mm}$. (b) Varied bunch length $\sigma$ for fixed taper length $L$ with $d=6 \mathrm{~mm}, b=3 \mathrm{~mm}, L=6.25 \mathrm{~mm}$, and $g=100 \mathrm{~mm} \quad($ red dots $) ; \quad d=50 \mathrm{~mm}, \quad b=6.25 \mathrm{~mm}, L=$ $50 \mathrm{~mm}$, and $\mathrm{g}=50 \mathrm{~mm}$ (green dots). large to assure longitudinal scaling (17) is accurately satisfied. We see that for $s / d \alpha \lesssim 0.3$, there is approximate agreement between the curves corresponding to different values of $b / d$, illustrating the weak dependence on the ratio $b / d$ in the approximate scaling relation (11).

The scaled loss factor $k_{\text {loss }} / k_{\text {loss }}^{\text {opt }}$ as a function of $\sigma / d \alpha$ for several tapered collimators is illustrated in Fig. 9. Good agreement is found with Eq. (12) for $\sigma / d \alpha \lesssim 0.15$.

\section{FURTHER DISCUSSION OF LOSS FACTOR}

In a previous paper [10], we presented a phenomenological formula approximating the loss factor of an axisymmetric collimator,

$$
k_{\mathrm{loss}} / k_{\mathrm{loss}}^{\mathrm{opt}} \cong\left[\frac{2}{\pi} \arctan \left(\frac{0.2 d^{2}}{\sigma L}\right)\right]^{2} .
$$

This formula is expected to hold for small $\sigma \ll b$ and large $d / b$. In Fig. 10, we present the data from Fig. 9(a), but now we plot $k_{\text {loss }} / k_{\text {loss }}^{\text {opt }}$ versus $L \sigma / d^{2}$.

We note that for small $\sigma \ll b$, large $d / b$, and large taper length $L$, the loss factor is well approximated by the asymptotic relation,

$$
\frac{k_{\text {loss }}}{k_{\text {loss }}^{\text {opt }}} \approx 0.016 \frac{d^{4}}{\sigma^{2} L^{2}} .
$$

\section{CONCLUDING REMARKS}

In Refs. [3,4], Podobedov and Stupakov noted that the point-charge wakefield for a collimator can be written in the form of Eq. (1).

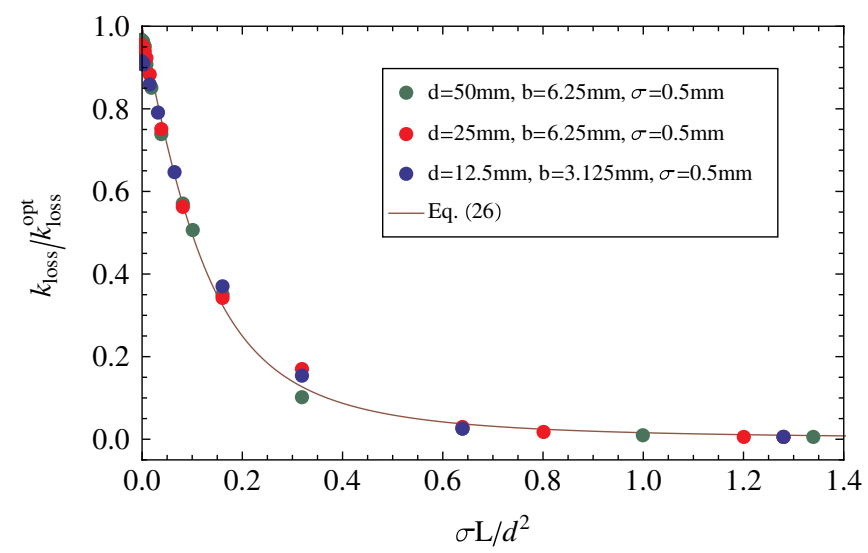

FIG. 10. We plot the ratio of the simulated loss factor (ECHO) to the loss factor in the optical regime $k_{\text {loss }} / k_{\text {loss }}^{\text {opt }}$ versus the dimensionless scaled length $L \sigma / d^{2}$ for the various tapered structures with fixed bunch length of $\sigma=0.5 \mathrm{~mm}$ and varied taper length for $d=50 \mathrm{~mm}$ and $b=6.25 \mathrm{~mm}$ (green dots), $d=$ $25 \mathrm{~mm}$ and $b=6.25 \mathrm{~mm}$ (red dots), and $d=12.5 \mathrm{~mm}$ and $b=$ $3.125 \mathrm{~mm}$ (blue dots). The inner length is $g=10 \mathrm{~mm}$. The dark red curve is given by Eq. (26). 
In this paper, we used the ECHO code $[1,2]$ to carry out an extensive study of the dependence of the wakefield on the transverse geometric parameters. For a tapered collimator, we have shown that the short-range wakefield, for $s / d \alpha \lesssim 0.3$ and $\alpha \lesssim \pi / 3$, is well approximated by Eq. (11).

Having factored out the $\log (d / b)$ term, we have found that in the range $1.5 \preceq \frac{d}{b} \preceq 6$ of Eq. (8), the scaling function $f$ depends only weakly on $b / d$.

For small taper angle, $\alpha \lesssim \pi / 4$, the ECHO calculations confirm that the longitudinal scaling [9] relation (17) is accurately satisfied, as shown in Eq. (17).

The approximation of Eq. (11) properly satisfies Eq. (17) for small taper angle. The longitudinal scaling is broken for angles large compared with $\pi / 4$. We have found that using the taper angle $\alpha$ in Eq. (11), rather than its tangent, allows Eq. (11) to remain a good approximation for larger angles $(\alpha \sim \pi / 3)$, beyond the regime where Eq. (17) is satisfied. As the taper angle increases further, Eq. (11) becomes less accurate, and the function $f$ begins to depend on the angle $\alpha$ itself.

The case of a step collimator $(\alpha=\pi / 2)$ is quite interesting. For the parameters that we have considered, its wakefield is well approximated by

$$
w^{\text {step }}(s ; b, d, g, L) \cong \frac{c Z_{0}}{\pi} \log \left(\frac{d}{b}\right)\left[\delta(s)-\frac{1}{d} F^{\text {step }}\left(\frac{s}{d}, \frac{b}{d}\right)\right],
$$

where the dependence on $b / d$ is very weak for $s / d>0.5$ (see Fig. 3). The very weak dependence of $F^{\text {step }}$ on $b / d$ is quite remarkable, and we did not anticipate this simple behavior.

\section{ACKNOWLEDGMENTS}

We wish to thank B. Podobedov for introducing us to Refs. [3,4] and for clarifying discussions on the work reported in this paper. This work was supported by Department of Energy Contract No. DE-AC0298CH10886.

\section{APPENDIX}

In Fig. 6, we plotted $d \alpha U^{\sigma_{0}}$ versus $s / d \alpha$. Here, in Fig. 11, we present a plot of $[d(d-b) / L] U^{\sigma_{0}}$ versus $L s /[d(d-b)]$. Comparison of these two figures illustrates the improvement for larger angles obtained by replacing $\tan \alpha$ by the angle $\alpha$ in transitioning from Eq. (21) to

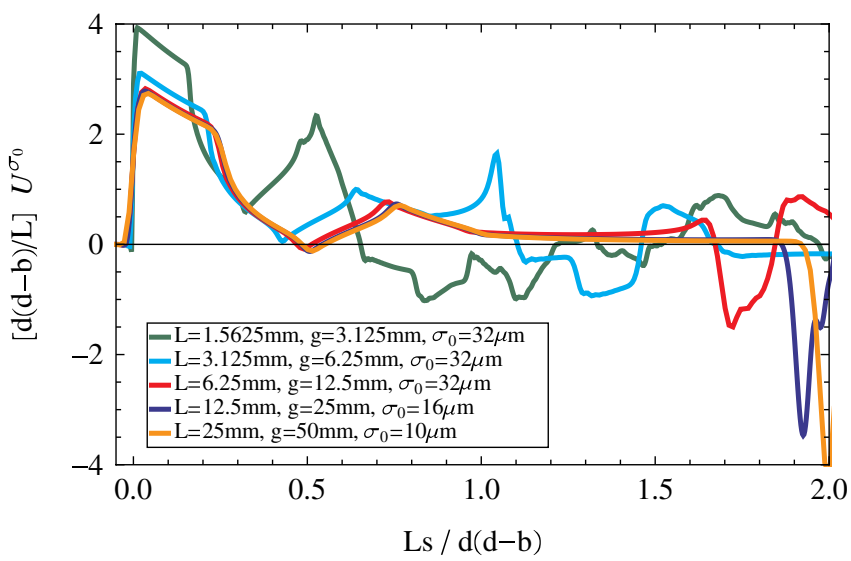

FIG. 11. For tapered collimator, we plot $[d(d-b) / L] U^{\sigma_{0}}$ versus $L s /[d(d-b)]$ for $d=6 \mathrm{~mm}, b=3 \mathrm{~mm}$ and $g=$ $3.12 \mathrm{~mm}, L=1.56 \mathrm{~mm}, \sigma_{0}=32 \mu \mathrm{m}$ (green); $g=6.25 \mathrm{~mm}$, $L=3.12 \mathrm{~mm}, \sigma_{0}=32 \mu \mathrm{m}$ (aqua); $g=12.5 \mathrm{~mm}, L=6.25$, $\sigma_{0}=32 \mu \mathrm{m}$ (red); $g=25 \mathrm{~mm}, L=12.5 \mathrm{~mm}, \sigma_{0}=16 \mu \mathrm{m}$ (blue); and $g=50 \mathrm{~mm}, L=25 \mathrm{~mm}, \sigma_{0}=8 \mu \mathrm{m}$ (orange).

Eq. (22). Of course, the scaling in Fig. 11 totally fails for $L \rightarrow 0$, while that in Fig. 6 still has some approximate validity as $\alpha \rightarrow \pi / 2$.

[1] I. Zagorodnov and T. Weiland, Phys. Rev. ST Accel. Beams 8, 042001 (2005).

[2] I. Zagorodnov, Phys. Rev. ST Accel. Beams 9, 102002 (2006).

[3] B. Podobedov and G. Stupakov, in Proceedings of the 2011 Particle Accelerator Conference, NY, USA (IEEE, New York, 2011), p. 1825.

[4] B. Podobedov and G. Stupakov, LER-2011, Heraklion, Greece (2011) [http://lowering2011.web.cern.ch/ lowering2011/].

[5] G. Stupakov, K. L. F. Bane, and I Zagorodnov, Phys. Rev. ST Accel. Beams 10, 054401 (2007).

[6] S. Heifets and S. Kheifets, Rev. Mod. Phys. 63, 631 (1991).

[7] H. Okamoto, S. Jiang, and R. Gluckstern, Phys. Rev. E 50, 1501 (1994).

[8] B. Zotter and S. Kheifets, Impedances and Wakes in HighEnergy Particle Accelerators (World Scientific Publishing Co., Singapore, 1997).

[9] G. Stupakov, K. L. F. Bane, and I Zagorodnov, Phys. Rev. ST Accel. Beams 14, 014402 (2011).

[10] A. Blednykh and S. Krinsky, Phys. Rev. ST Accel. Beams 13, 064401 (2010). 